\title{
Product Rearrangement from Altering a Single Residue in the Rice syn-Copalyl Diphosphate Synthase
}

\author{
Kevin C. Potter, ${ }^{\dagger} \S$ Meirong Jia, ${ }^{\dagger}$ Young J. Hong, ${ }^{\ddagger}$ Dean Tantillo, ${ }^{\dagger}$ and Reuben J. Peters ${ }^{* \dagger}$ \\ ${ }^{\dagger}$ Roy J. Carver Department of Biochemistry, Biophysics and Molecular Biology, Iowa State University, Ames, Iowa 50011, United \\ States \\ ${ }^{\ddagger}$ Department of Chemistry, University of California, Davis, California 95616, United States
}

Supporting Information

ABSTRACT: Through site-directed mutagenesis targeted at identification of the catalytic base in the rice (Oryza sativa) syncopalyl diphosphate synthase OsCPS4, changes to a single residue (H501) were found to induce rearrangement rather than immediate deprotonation of the initially formed bicycle, leading to production of the novel compound syn-halimadienyl diphosphate. These mutational results are combined with quantum chemical calculations to provide insight into the underlying reaction mechanism.

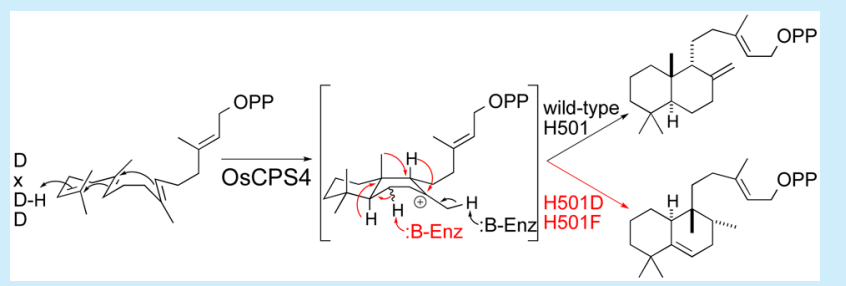

Scheme 1. Cyclization of GGPP (1) to syn-CPP (2) or synHPP (3) by Wild-Type or H501 Mutants of OsCPS4,

Respectively

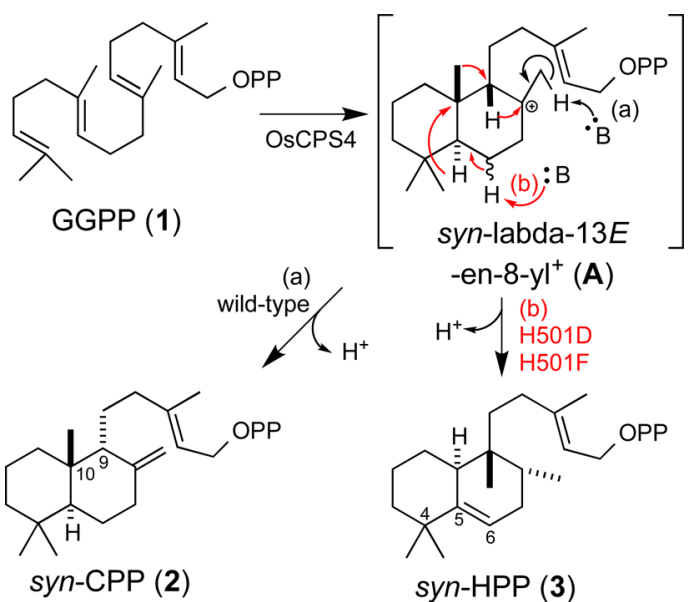

which can be rearranged by sequential 1,2-shifts of hydride and methyl groups, with the $\mathrm{C} 10 \rightarrow \mathrm{C} 9$ methyl migration forming the halimadane skeletal structure and subsequent $\mathrm{C} 4 \rightarrow \mathrm{C} 5$ methyl migration forming the clerodane backbone (Scheme S1). ${ }^{1}$ The final carbocation seems to be invariably quenched by deprotonation, although this may be preceded by the addition of water, resulting in a hydroxylated product. Notably, while DTCs/CPSs are characterized by a highly conserved $(\mathrm{D}, \mathrm{E}) \mathrm{xDD}$ motif from which the middle aspartic acid acts as the catalytic acid, ${ }^{7}$ the catalytic base does not appear to be nearly as well conserved. This may be due in part to variation in

Received: January 19, 2016

Published: February 15, 2016 
positioning of the base, relative to the acid, underlying the production of different (stereo)-isomers of CPP, as well as the addition of water and/or rearrangement, by various DTCs.

The catalytic base for the plant CPSs involved in gibberellin phytohormone biosynthesis has been recently identified as dependent on a histidine-asparagine dyad that is specifically conserved in these ent-CPP producing DTCs. ${ }^{8}$ By contrast, the catalytic base in the DTC active site of the diterpene synthases involved in conifer resin acid biosynthesis has been identified as a very similarly positioned tyrosine-histidine dyad, which is specifically conserved in these normal CPP $(9 S, 10 S)$ producing enzymes., 10 In both cases, alanine substitution for the histidines leads to the addition of water, with the mutant DTCs yielding hydroxylated derivatives of CPP. ${ }^{8,9}$

Consistent with its closer phylogenetic relationship to other CPSs, OsCPS4 contains the histidine (H251) of the CPS catalytic base dyad, although the other position is a cysteine (C310) instead of asparagine. However, alanine substitution for this histidine did not eliminate activity, nor alter product outcome, with expression of the OsCPS4:H251A mutant in E. coli engineered to produce $\mathbf{1}$ via a modular metabolic engineering system ${ }^{11}$ still leading to the exclusive production of 2 (Figure S1). Similarly, alanine substitution for the cysteine also does not significantly alter activity (i.e., OsCPS4:C310A also produces 2, Figure S1). In addition, while substitution of aspartate for the catalytic histidine in the DTC active site of the diterpene synthases involved in conifer resin acid biosynthesis also leads to incorporation of water, ${ }^{9}$ such substitution in OsCPS4 simply reduces catalytic activity (i.e., OsCPS4:H251D produces only trace amounts of 2, Figure S1). Accordingly, OsCPS4 appears to use a novel general base, consistent with the unique stereochemistry of its product.

The identity of the catalytic base in OsCPS4 was further investigated by protein structure modeling. A model was constructed on the basis of the most closely related known crystal structure, that of the CPS from Arabidopsis thaliana (AtCPS), ${ }^{12,13}$ using the SWISS-MODEL server. ${ }^{14-17}$ The previously identified catalytic base groups both include histidines, which were found across the active site from the DxDD motif. In addition to $\mathrm{H} 251$, two other similarly positioned histidines ( $\mathrm{H} 275$ and $\mathrm{H} 501)$ are present in the modeled OsCPS4 active site (Figure 1A).

Notably, while substitution of either alanine or aspartate for $\mathrm{H} 275$ did not alter product outcome, and only slightly reduced overall yield (Figure S2), such substitutions for $\mathrm{H} 501$ had a clear effect (Figure 2A-C). The OsCPS4:H501A mutant was found to exhibit both reduced yield and a mixture of $\mathbf{2}$ with an unidentified product whose mass spectra indicated that it was not a hydroxylated version of $\mathbf{2}$ (i.e., from incorporation of water), as the molecular weight of the dephosphorylated derivative appeared to be 290 (equivalent to that for 2; Figure 2D). With the OsCPS4:H501D mutant, although 2 is still observed and the overall yield lags that observed with the wildtype enzyme (i.e., substantial amounts of $\mathbf{1}$ are still present), this compound was produced in much higher amounts, enabling purification and structural analysis of the dephosphorylated derivative by NMR (Table S1, Figures S3-S5). The compound was found to be a halima-5,13E-dien-15-ol whose stereochemical configuration indicates its derivation from rearrangement of syn-labda-13E-en-8-yl ${ }^{+}$(A, for structure see Scheme 1). Accordingly, the enzymatic product is termed here syn-halima-5,13E-dienyl diphosphate (syn-HPP, 3). Given that substitution of phenylalanine and, especially, tyrosine for the

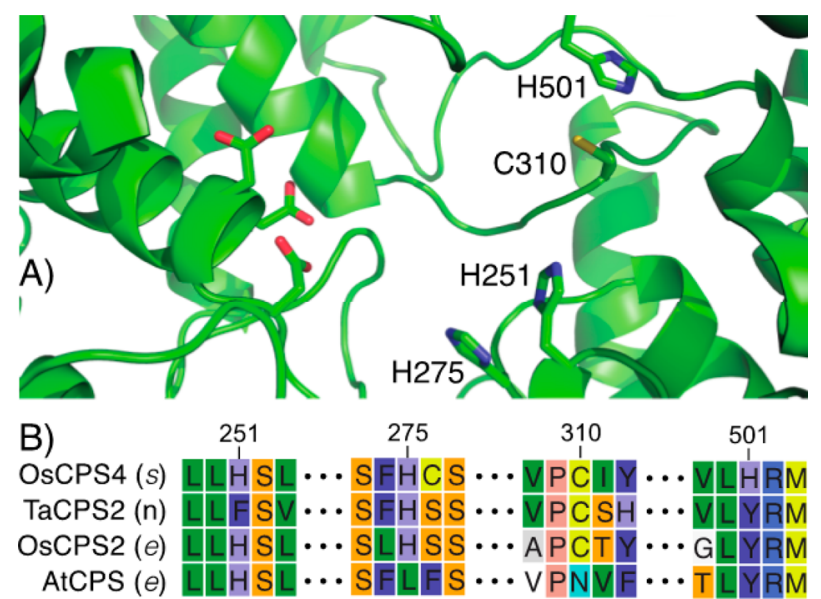

Figure 1. OsCPS4 active site. (A) From modeled structure, shown in cartoon format with stick representation of the side chains of the aspartate residues of the DxDD motif (on the left) and the targeted histidines and cysteine (on the right), created using PyMol. ${ }^{18}$ (B) Conservation of targeted residues in selected CPSs discussed in text, with stereochemistry of CPP products as indicated (s, syn; n, normal; $e$, ent), numbering as in panel A (i.e., from OsCPS4).
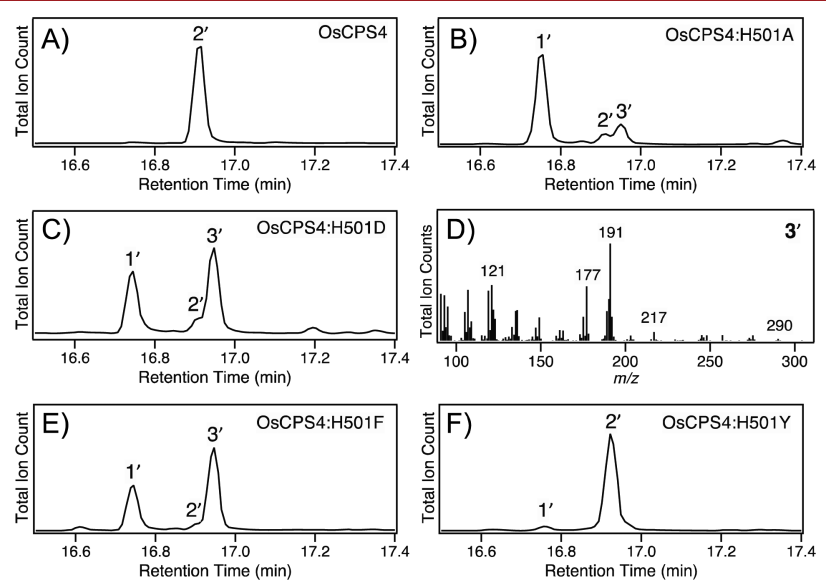

Figure 2. Effect of various substitutions for $\mathrm{H} 501$ on OsCPS4 product outcome. GC-MS chromatograms of the dephosphorylated products extracted from OsCPS4 (wild-type and indicated mutants) in E. coli engineered to produce 1 , along with mass spectra for the observed novel product (numbering as in text, with prime ' notation used to indicate that these are dephosphorylated derivatives).

catalytic histidine in AtCPS has recently been shown to result in product rearrangement, ${ }^{19}$ analogous mutants were constructed for OsCPS4. Strikingly, these had contrasting effects, with phenylalanine substitution exhibiting the expected production of 3, with improved yield relative to the aspartate mutant (both overall and as an increased proportion of the product mix), while tyrosine substitution does not alter the production of 2 , which is observed in good yield, with very little 1 left (Figure 2E and F).

Interestingly, H501 occupies a position that is otherwise invariably conserved as a tyrosine in plant DTCs, highlighting its functional role in the stereochemically unique reaction catalyzed by OsCPS4. By contrast, the position occupied by $\mathrm{H} 275$ is more variable. While this residue is generally an (iso)leucine in most plant DTCs, histidine can be found at this position in other closely related CPSs that produce different stereoisomers of CPP (Figure 1B), both the ent-CPP producing 
OsCPS2 from rice ${ }^{20}$ and the normal CPP producing TaCPS2 from wheat (Triticum aestivum). ${ }^{21}$ However, although it is possible that $\mathrm{H} 501$ acts as the catalytic base, the observed mixture of effects on product outcome from various substitutions differs from those previously observed for the catalytic bases in other DTCs, ${ }^{8,9,19}$ which seems to preclude clear assignment of the role H501 plays in shaping product outcome.

It is, further, not immediately evident why 3 is the observed alternative product. This is formed via rearrangement of $\mathbf{A}$ to a halimadienyl $^{+}$intermediate, with no $\mathrm{C} 4 \rightarrow \mathrm{C} 5$ methyl migration to the clerodane backbone observed. Production of 3 contrasts with the effect of aromatic substitution for the catalytic histidine in AtCPS, where such further rearrangement is observed, with removal of the same proton originally added to initiate the reaction, presumably by returning to the same aspartate that acted as the catalytic general acid. ${ }^{19}$ However, quantum chemical calculations indicated that, at least with the relevant ent-labda-13E-en-8-yl ${ }^{+}$intermediate, this last 1,2 methyl shift is much more energetically difficult than the preceding 1,2-shifts. Both the transition state structures and resulting tertiary carbocation intermediates for these 1,2-shifts are within $\sim 7 \mathrm{kcal} \mathrm{mol}^{-1}$ of the initial ent-labda-13E-en-8-yl ${ }^{+}$ intermediate, while the final $\mathrm{C} 4 \rightarrow \mathrm{C} 5$ methyl shift has an energetic barrier of $\sim 14 \mathrm{kcal} \mathrm{mol}^{-1}$ and the resulting entcleroda-13E-en-4-yl ${ }^{+}$intermediate is significantly higher in energy than the other intermediates. ${ }^{19}$ Moreover, the introduced aromatic group is relatively distant and, thus, seems unlikely to drive this last methyl shift, such that production of the clerodane skeletal structure by these AtCPS mutants is then likely driven by the lack of suitable general bases for deprotonation of any of the other, relatively more stable preceding intermediates.

Previous semiempirical quantum chemical calculations suggested that the various tertiary carbocation intermediates in rearrangement of syn-labda-13E-en-8-yl ${ }^{+}$(A) are all of similar energy (spanning a range of only $5 \mathrm{kcal} \mathrm{mol}^{-1}$ ). Specifically, the 9- $\mathrm{yl}^{+} \mathbf{B}$ (see Figure 3) formed by initial 1,2-hydride migration is

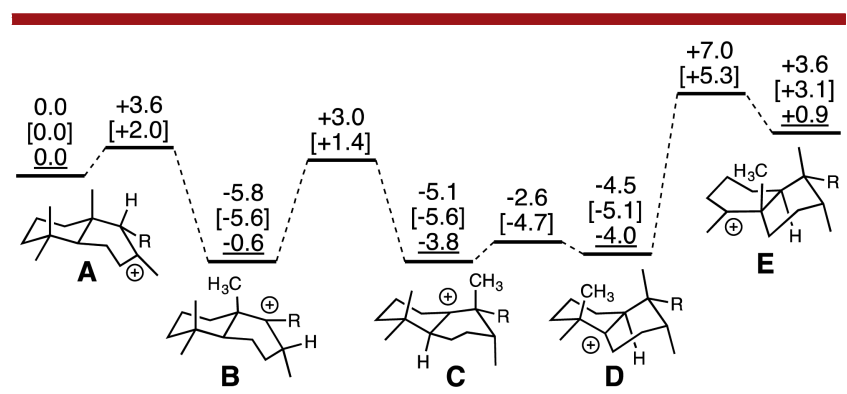

Figure 3. Energies of carbocation minima and transition-state structures involved in hydride and methyl shifts en route to 3 . Relative energies (B3LYP/6-31+G(d,p) in normal text, mPW1PW91/ $6-31+\mathrm{G}(\mathrm{d}, \mathrm{p}) / / \mathrm{B} 3 \mathrm{lYP} / 31+\mathrm{G}(\mathrm{d}, \mathrm{p})$ [in brackets], and PM3 from ref 22 underlined) are shown in $\mathrm{kcal} \mathrm{mol}^{-1}$; in this model, $\mathrm{R}=$ $\left(\mathrm{CH}_{2}\right)_{2} \mathrm{C}(\mathrm{Me})=\mathrm{CHCH}_{2} \mathrm{OPO}_{3} \mathrm{H}_{2}$.

predicted to be $<1 \mathrm{kcal} \mathrm{mol}^{-1}$ lower in energy than $\mathbf{A}$, and the syn-halima-13E-en-10-yl ${ }^{+} \mathrm{C}$ formed by subsequent 1,2-methyl migration is predicted to be $4 \mathrm{kcal} \mathrm{mol}^{-1}$ lower in energy than A and essentially isoenergetic with the syn-halimad-13E-en-5-yl ${ }^{+}$ D formed by subsequent 1,2-hydride migration, while the syncleroda-13E-en-4-yl ${ }^{+}$E formed by the final 1,2-methyl migration is predicted to be only $1 \mathrm{kcal} \mathrm{mol}^{-1}$ higher in energy than $\mathbf{A}^{22}$ Here quantum chemical calculations, using density functional theory (DFT) methods previously applied to characterization of terpene carbocation rearrangements (see Supporting Information for details $)^{23,24}$ and with proven utility $^{25}$ were used to investigate not only these intermediates but also the corresponding transition state structures for their interconversion (Figure 3, numbers in normal text and brackets; see also Figure S6). This analysis was broadly supportive of the previously reported results, although the relative stability of $\mathbf{A}$ appears to have been overestimated by the utilized semiempirical method (Figure 3). In addition, the barrier for the final 1,2-methyl shift is predicted to be smaller than that found previously for rearrangement of ent-labda-13Een- $8-\mathrm{yl}^{+},{ }^{19}$ both the barrier from $\mathrm{D}$ (lower by $\sim 4 \mathrm{kcal} \mathrm{mol}^{-1}$ ) and the overall barrier from $\mathrm{A}$ (lower by $\sim 12 \mathrm{kcal} \mathrm{mol}^{-1}$ ). ${ }^{26}$ Accordingly, there is not a large energetic barrier to this final 1,2-methyl shift, indicating that there is a suitably positioned general base in the OsCPS4 active site for deprotonation of $\mathbf{D}$ (note that this would not interfere with initial bicyclization, as this does not proceed via a corresponding $5-\mathrm{yl}^{+}$intermediate), which alleviates any need to undergo full rearrangement to $\mathbf{E}$ to allow for removal of the initiating proton, unlike what is observed upon aromatic replacement of the catalytic histidine in AtCPS. ${ }^{19}$ Identification of this otherwise "silent" general base, as well as elucidation of the exact role of H501, will be the subject of future investigation and may require determination of a high-resolution structure for OsCPS4.

Regardless of the exact mechanism underlying this observed change in product outcome, it is notable that these OsCPS4:H501 mutants appear to represent the first identified syn-HPP synthase, which then offers access to derived natural products. For example, 3 is likely to be the precursor to various labdane-related diterpenoids isolated from Vitex agnus and Vitex rotundifolia, which are Chinese medicinal plants that have been widely used in Korea, China, and Japan for the treatment of inflammation, headache, migraine, chronic bronchitis, eye pain, and gastrointestinal infections. ${ }^{27,28}$ Finally, these studies further illustrate the plasticity of DTCs, which underlies the expansive evolution of the labdane-related diterpenoid superfamily of natural products.

\section{ASSOCIATED CONTENT}

\section{Supporting Information}

The Supporting Information is available free of charge on the ACS Publications website at DOI: 10.1021/acs.orglett.6b00181.

Description of experimental methods; GC-MS chromatograms for other OsCPS4 mutants described; NMR chemical shift data and spectra for $3^{\prime}$; Details of quantum chemical calculations (PDF)

\section{AUTHOR INFORMATION}

\section{Corresponding Author}

*E-mail: rjpeters@iastate.edu.

\section{Present Address}

${ }^{\S}$ Department of Biology, University of North Carolina, Chapel Hill, North Carolina 27599, United States.

\section{Notes}

The authors declare no competing financial interest. 


\section{ACKNOWLEDGMENTS}

This work was supported by grants from the NIH (GM076324 to R.J.P.) and NSF (CHE-1361807 to D.J.T.).

\section{REFERENCES}

(1) Peters, R. J. Nat. Prod. Rep. 2010, 27, 1521-1530.

(2) Zi, J.; Mafu, S.; Peters, R. J. Annu. Rev. Plant Biol. 2014, 65, 259286.

(3) Xu, M.; Hillwig, M. L.; Prisic, S.; Coates, R. M.; Peters, R. J. Plant J. 2004, 39, 309-318.

(4) Xu, M.; Galhano, R.; Wiemann, P.; Bueno, E.; Tiernan, M.; Wu, W.; Chung, I. M.; Gershenzon, J.; Tudzynski, B.; Sesma, A.; Peters, R. J. New Phytol. 2012, 193, 570-5.

(5) Kato-Noguchi, H.; Peters, R. J. J. Chem. Ecol. 2013, 39, 175-185.

(6) Schmelz, E. A.; Huffaker, A.; Sims, J. W.; Christensen, S. A.; Lu, X.; Okada, K.; Peters, R. J. Plant J. 2014, 79, 659-678.

(7) Prisic, S.; Xu, J.; Coates, R. M.; Peters, R. J. ChemBioChem 2007, 8, 869-874.

(8) Potter, K.; Criswell, J.; Peters, R. J. Angew. Chem., Int. Ed. 2014, 53, 7198-7202.

(9) Criswell, J.; Potter, K.; Shephard, F.; Beale, M. B.; Peters, R. J. Org. Lett. 2012, 14, 5828-5831.

(10) Mafu, S.; Potter, K. C.; Hillwig, M. L.; Schulte, S.; Criswell, J.; Peters, R. J. Chem. Commun. (Cambridge, U. K.) 2015, 51, 13485-7.

(11) Cyr, A.; Wilderman, P. R.; Determan, M.; Peters, R. J. J. Am. Chem. Soc. 2007, 129, 6684-6685.

(12) Köksal, M.; Hu, H.; Coates, R. M.; Peters, R. J.; Christianson, D. W. Nat. Chem. Biol. 2011, 7, 431-3.

(13) Köksal, M.; Potter, K.; Peters, R. J.; Christianson, D. W. Biochim. Biophys. Acta, Gen. Subj. 2014, 1840, 184-90.

(14) Arnold, K.; Bordoli, L.; Kopp, J.; Schwede, T. Bioinformatics 2006, 22, 195-201.

(15) Biasini, M.; Bienert, S.; Waterhouse, A.; Arnold, K.; Studer, G.; Schmidt, T.; Kiefer, F.; Cassarino, T. G.; Bertoni, M.; Bordoli, L.; Schwede, T. Nucleic Acids Res. 2014, 42, W252-8.

(16) Guex, N.; Peitsch, M. C.; Schwede, T. Electrophoresis 2009, 30 (Suppl. 1), S162-73.

(17) Kiefer, F.; Arnold, K.; Künzli, M.; Bordoli, L.; Schwede, T. Nucleic Acids Res. 2009, 37, D387-92.

(18) Stofer Vogel, B.; Wildung, M. R.; Vogel, G.; Croteau, R. J. Biol. Chem. 1996, 271, 23262-23268.

(19) Potter, K. C.; Zi, J.; Hong, Y. J.; Schulte, S.; Malchow, B.; Tantillo, D. J.; Peters, R. J. Angew. Chem., Int. Ed. 2016, 55, 634-638.

(20) Prisic, S.; Xu, M.; Wilderman, P. R.; Peters, R. J. Plant Physiol. 2004, 136, 4228-4236.

(21) Wu, Y.; Zhou, K.; Toyomasu, T.; Sugawara, C.; Oku, M.; Abe, S.; Usui, M.; Mitsuhashi, W.; Chono, M.; Chandler, P. M.; Peters, R. J. Phytochemistry 2012, 84, 40-46.

(22) Eguchi, T.; Dekishima, Y.; Hamano, Y.; Dairi, T.; Seto, H.; Kakinuma, K. J. Org. Chem. 2003, 68, 5433-8.

(23) Tantillo, D. J. Nat. Prod. Rep. 2011, 28, 1035-53.

(24) This is part 12 in our series on quantum calculations on diterpene-forming carbocation rearrangements; for part 11 , see ref 19 . (25) Zu, L.; Xu, M.; Lodewyk, M. W.; Cane, D. E.; Peters, R. J.; Tantillo, D. J. J. Am. Chem. Soc. 2012, 134, 11369-71.

(26) Conversion to an alternative conformer of $\mathrm{D}$ leads to a barrier that is lower still (by an additional $1.2 \mathrm{kcal} \mathrm{mol}^{-1}$ ), but whether the necessary conformational change could occur in the active site is not clear. See Supporting Information for details.

(27) Lee, C.; Lee, J. W.; Jin, Q.; Lee, H. J.; Lee, S. J.; Lee, D.; Lee, M. K.; Lee, C. K.; Hong, J. T.; Hwang, B. Y. Bioorg. Med. Chem. Lett. 2013, $23,6010-4$.

(28) Ono, M.; Yamasaki, T.; Konoshita, M.; Ikeda, T.; Okawa, M.; Kinjo, J.; Yoshimitsu, H.; Nohara, T. Chem. Pharm. Bull. 2008, 56, $1621-4$. 